\title{
EL "PLAN DE LAS INMEDIACIONES" DEL OBSERVATORIO ASTRONÓMICO: UN PROYECTO DESAPARECIDO DE JUAN DE VILLANUEVA
}

\author{
POR \\ LUIS RAMÓN - LACA MĖNÉNDEZ DE LUARCA \\ Real Jardín Botánico, C.S.I.C.
}

\begin{abstract}
This paper reconstructs the "plan de las inmediaciones" ("project for the surroundings") hypothetically composed by Juan de Villanueva for Madrid Observatorio Astronómico, today disappeared. The data emanated from the drawing dedied to the Observatorio by Isidro Velázquez, Villanueva's best disciple, as well as from the parcel, allow to draw the perfect integration between the building and the surrounding landscape. Because of a number of circumstances two important elements are losten, the original access building and the great 25 feet telescope. Nevertheless, the restoration of the scene conceived by Villanueva, and later drawn by Velázquez, is still perfectly possible.
\end{abstract}

\section{INTRODUCCIÓN}

El Observatorio Astronómico es sin duda uno de los mejores ejemplos de arquitectura neoclásica con que cuenta la ciudad de Madrid. Del proyecto original de Juan de Villanueva (1739-1811) se conservan la planta y el alzado del edificio, mientras que un "plan de las inmediaciones", supuestamente elaborado por el mismo arquitecto, se encuentra hoy en paradero desconocido. A lo largo de este trabajo voy a tratar de reconstruir dicho plan, basándome para ello en la aguada de Isidro Velázquez conservada en el Museo Municipal de Madrid, así como en las evidencias derivadas del estudio del solar del propio Observatorio. ${ }^{1}$ (Fig. 1)

El edificio del Observatorio es bien conocido, habiéndosele dedicado numerosos trabajos, a pesar de lo cual, y debido a las modificaciones sustanciales ocurridas en aquellas

\footnotetext{
${ }^{1}$ Isidro Velázquez (1765-1829) fue uno de los más allegados discípulos de Villanueva, y como tal aparece citado el primero entre los legatarios de los bienes en el testamento de aquel:

«A D. Isidro Velazquez, discípulo de dicho Señor Villanueva, la caja de tintas y colores $q^{\mathrm{e}}$. conservava el difunto con toda su servidumbre, para que la use así en su nombre, y que le encomiende a Dios.» Archivo de Protocolos, «Testamento de Juan de Killanueva», 18 de septiembre de 1811, fos. 379r-380v.
} 
"inmediaciones", no alcanza la dimensión que cabe imaginar con arreglo al referido proyecto. ${ }^{2}$ El conjunto del Observatorio se encuentra hoy sumamente desfigurado por la acumulación de material de derribo y construcciones de escaso interés. Los jardines presentan un penoso estado, con numerosos árboles mutilados debido a podas antiguas mal realizadas, así como abundantes restos constructivos diseminados por doquier. En realidad esta situación ha sido la tónica general a lo largo de toda la historia del Observatorio, y ya en 1865 la misma Junta de la Academia de San Fernando recomendaba "despojar" al Observatorio "de todo cuanto se le había agregado":

«Junta del dia 22 de Junio 1865

Se ocupó del expediente remitido por la Dirección General de Instrucción publica para reformar el templete del edificio que ocupa el Observatorio, y de acuerdo con el ponente Dn. Anibal Alvarez no autorizó la expresada reforma bajo ningun concepto, por tratarse de una joya del arte moderno, y que si alguna reforma cabe sería despojarle de todo cuanto se le ha agregado.

Es verdaderamente terrible que personas tan ilustradas y cultas como son las que se hallan al frente del Observatorio, hayan concebido la idea de desfigurar este precioso edificio, destruyendo su cupula, con lo cual se le quita todo su caracter, y que si no reune las condiciones que el desarrollo y adelanto de la ciencia reclama, no es suficiente razon para mutilar tan precioso edificio y medios habra para obtener el resultado que se busca...» $»^{3}$

En efecto, debido a una serie de circunstancias que analizaremos a lo largo de este trabajo, el proyecto original fue modificado notablemente, sobre todo en lo que se refiere a los accesos originales al edificio. De modo paradójico, la entrada al edificio se realiza hoy por la puerta de atrás, mientras que el pórtico principal desempeña el papel de fachada trasera. Todo ello debe atribuirse al enterramiento de la construcción original de acceso bajo sucesivas capas de materiales de derribo. Es imposible tener una perspectiva general del conjunto, y sólo desde un punto muy concreto de la Estación de Atocha puede verse parcialmente el edificio. Hace ya tiempo que un conocido crítíco de arquitectura afirmó que se había enviado el Observatorio "al trastero de la ciudad."

Cualquiera que se acerque a visitar el solar del Observatorio comprobará que el tono pesimista de estos apuntes previos corresponde plenamente a la realidad, queriendo reclamar con estas líneas mayor atención para una obra que bien la merece. La única razón para el optimismo estriba en el hecho de que la recuperación de este desaparecido proyecto es aún perfectamente posible.

\footnotetext{
${ }^{2}$ Sobre el Observatorio ver: CHUECA GOITIA, F. y MIGUEL, C. DE: La vida y las obras del arquitecto Juan de Villanueva, S. N., Madrid, 1949. KUBLER, G.: Arquitectura de los siglos XVII y XVIII. Ars Hispaniae, Plus-Ultra, Madrid, 1957. FERNÁNDEZ ALBA, A.: El Observatorio Astronómico de Madrid/Juan de Villanueva, arquitecto, Xarait, Madrid, 1979. MOLEÓN GAVILANES, P.: La arquitectura de Juan de Villanueval El proceso del proyecto, C.O.A.M., Madrid, 1988. MOLEÓN GAVILANES, P.: «El edificio Villanueva del Real Observatorio Astronómico de Madrid», en Doscientos años del Observatorio Astronómico de Madrid, Asociación de Amigos del Observatorio Astronómico, Madrid, 1992, 27-68.

${ }^{3}$ Archivo de la Academia de Bellas Artes de San Fernando, 43-1/2.

${ }^{4}$ «En su tiempo [el Observatorio] se vería al final de la perspectiva del Paseo del Prado, y sería un regalo para los ojos Hoy no se ve desde ninguna parte, rodeado como está de bloques de casas. Se le ha enviado al trastero de la ciudad.» CHUECA GOITIA, F.: «Juan de Villanueva: su significación en la historia de la arquitectura española», en Juan de Villanueva/ Arquitecto (1739-1811), Museo Municipal, Ayuntamiento de Madrid-Delegación de Cultura, 1982, 45.
} 


\section{LA COMPLEJA HISTORIA DEL OBSERVATORIO ASTRONÓMICO}

Entre 1790 y 1795 Villanueva dirigió la construcción del Observatorio en un alto situado en el llamado Campo de San Blas.(Fig. 2) En un informe elaborado en 1819 por el entonces Arquitecto Mayor hay referencia a un proyecto de diseño general, obra de Villanueva, lamentablemente perdido:

«Cuando se construyó el Real Observatorio de San Blas, se formó plan de todas las inmediaciones por el Arquitecto don Juan de Villanueva en el que estaba incluida la reedificación de la Ermita citada, que debía hacer frente y medio con dicho edificio del Observatorio, cuyo plan fue aprobado en tiempo del Señor Floridablanca por S. M. en todas sus partes.» ${ }^{5}$

El interés demostrado por el arquitecto madrileño en relación a los terrenos situados al sur del Observatorio hace pensar que dicho proyecto incluiría un acceso desde la parte baja de Atocha, que desgraciadamente no fue posible llevar a cabo debido a que en los referidos terrenos, propiedad de particulares, se levantaban diversas construcciones.

A pesar de las reiteradas recomendaciones de traslado por parte de Villanueva, dichos terrenos no fueron enajenados nunca. Veamos rápidamente cuales fueron las circunstancias de este proceso. El 26 de junio de 1791, Diego Bravo Ximenez de Zisneros, patrono de la Ermita de San Blas, solicitó licencia municipal de obras para "fabricar de nueba Planta el Juego de pelota contiguo a dha Hermita [de San Blas] y Casa y reparar estas." El 4 de julio del mismo año Villanueva, en su doble condición de Arquitecto Mayor de Madrid y encargado de la obra del Observatorio, propuso el traslado del juego de pelota y la Ermita de San Blas a un lugar más adecuado, con el fin de habilitar "una decente y bien decorada entrada a aquel nuevo Edificio [el Observatorio]." A pesar de que Villanueva se basaba en una orden del Conde de Floridablanca, en aquel entonces Primer Ministro, los propietarios de la Ermita consiguieron su anulación, ante lo cual manifestó el arquitecto su temor de que se elevara "en aquella parte un feo Edificio particular" que causara "embarazo á la vista de otro publico mas util, costoso y de mejor decoracion."

Doscientos años después estas palabras resultan proféticas, ya que como Villanueva temía, la decisión final de conceder el permiso solicitado por los demandantes iba a perjudicar notablemente al Observatorio. Si contemplamos las casas de vecinos que se levantan hoy al sur del edificio la advertencia, nunca llevada a efecto, de que "si en lo sucesivo se viese que estorba la vista del Observatorio Astronómico, se tomaría entonces providencia" resulta cuando menos ridícula. ${ }^{6}$

A estas complicaciones hay que añadir además las derivadas de la Guerra de Independencia, durante la cual el Observatorio sufrió graves daños que obligaron a una importante reparación, no concluida hasta $1847 .{ }^{7}$ Fue necesario reparar la tapia que separaba el Observatorio del Retiro, dañada durante la fortificación de la vecina Fábrica de la China llevada a cabo por el ejército francés. ${ }^{8}$ La Ermita fue finalmente demolida en 1812, sin que por ello variara el límite sur de la propiedad. Las peticiones económicas para costear los gastos ocasionados por obras fueron frecuentes durante los años posteriores a la Guerra. Así, por ejemplo, en 1819 el arquitecto Antonio López Aguado solicitó 900 arrobas de plomo (aprox. 10 Tm) "para evitar

\footnotetext{
${ }^{5}$ SIMÓN PALMER, M. DEL C.: «La Ermita y el Cerrillo de San Blas», 122-123, en Anales del Instituto de Estudios Madrileños IX, Madrid, C.S.I.C. (1973), 117-126.

${ }^{6}$ MOLEÓN GAVILANES, P.: La arquitectura de Juan de Villanueva, apéndice 3.

${ }^{7}$ MADOZ, P.: Diccionario geográfico-estadístico-histórico, vol. X, Madrid, 1847, 816.

${ }^{8}$ CABAÑAS DIAZ, J. C.: «Historia de los terrenos del Observatorio Astronómico de Madrid», 71, en Doscientos años del Observatorio Astronómico de Madrid, Asociación de Amigos del Observatorio Astronómico, Madrid, 1992, 69-84.
} 
la ruina de aquel hermoso edificio", mientras que en 1829 se informaba nuevamente del estado de la obra del Observatorio:

«El Edificio del Observatorio está sin concluir y según lo informado á la Junta por D. Antonio Lopez Aguado en 16 de Sbre. último se necesitan unos 117.000 rs. para ejecutar la obra que falta.»’

La institución comenzó a funcionar por fin a partir de 1851, comprándose e instalándose el nuevo instrumental entre 1853 y $1858 .{ }^{10}$ Los terrenos del Observatorio no quedaron delimitados hasta 1853, aunque la posterior construcción de la antigua calle de Granada, hoy calle de Alfonso XII, modificaría una vez más los límites de la parcela.

\section{EL CUERPO DE ESCALERAS}

Como han observado otros autores, llama la atención en la citada aguada de Isidro Velázquez una construcción adosada al montículo sobre el que se levanta el edificio. Se trata del primitivo cuerpo de escaleras de acceso al edificio, cuya presencia aparece esbozada ya en la planta original del edificio.(Fig. 3) Corresponde al ingeniero técnico topógrafo J. C. Cabañas Diaz, a quien desgraciadamente me ha sido imposible localizar durante la realización de este trabajo, el mérito de haber sido el primero en subrayar la existencia de dicha construcción:

«Por otra parte y aunque no es misión de este trabajo, aprovecho para solicitar a quien tenga competencia para ello, que se busque la forma de sacar a la luz la primitiva entrada del Observatorio, la cual es también obra de Juan de Villanueva, y se encuentra enterrada al sur de éste.» ${ }^{11}$

El trabajo de este autor demuestra que un examen atento de la topografía resulta suficiente para asegurar la existencia de las susodichas escaleras. En efecto, el diseño extraño e irregular que hoy presenta el terreno alrededor del edificio se debe a modificaciones posteriores a su construcción, como puede comprobarse fácilmente consultando el preciso Plano parcelario de Madrid de 1872-74. ${ }^{2}$ (Figs. 4, 5 y 6) En esta época las curvas de nivel definen perfectamente el montículo, lo que significa que los 17 grandes bloques de granito existentes al sur del edificio corresponden al pretil de la citada construcción, tal y como aparece en la aguada de Velázquez.

La acumulación de material sobre el terreno tuvo que llevarse a cabo por tanto en época reciente, ya que a mediados del siglo XIX tanto el montículo como la caja de escaleras aparecen con claridad en diversos grabados.(Fig. 7) En 1915 la caja de escaleras sobresale parcialmente del terreno, mientras que en 1920 apenas si asoma bajo el pretil de granito una pequeña parte del paramento de ladrillo.

El enterramiento de este elemento resta al edificio gran parte de sentido, resultando su diseño sumamente revelador de la mentalidad del arquitecto madrileño. Como él mismo nos indica en su

\footnotetext{
${ }^{9}$ Archivo del Museo Nacional de Ciencias Naturales, "Real Observatorio Astronómico de Madrid", caja 1, carpetas 4 y 12.

${ }^{10}$ VERNET GINÉS, J.: «Cultura científica y astronómica en los siglos XVIII y XIX», en Astronomía y cartografía de los siglos XVIII y XIX, Comisión Quinto Centenario, Observatorio Astronómico Nacional, I.G.N., Madrid, 1987, 7.

${ }^{"}$ CABAÑAS DIAZ, J. C.: «Historia de los terrenos del Observatorio Astronómico de Madrid», 83-84.

${ }^{12}$ IBÁÑEZ E IBÁÑEZ DE IBERO, C.: Plano parcelario de Madrid, Instituto Geográfico y Estadístico, Madrid, 1872-74.
} 
Descripción del edificio del Rl. Museo, se proponía en aquella obra [del Museo] "hacer patente y visible parte de aquellas bellezas y grandiosidades que tenia vistas y observadas en las ruinas de la antigüedad y en los edificios de la Roma moderna." ${ }^{\prime 3}$ Lo que vale para el Museo puede decirse también del Observatorio, en el que el cuerpo de escaleras, con su característico aire egipcio, se convierte en un fascinante ejemplo del estilo neoclásico puro proclamado por Villanueva.(Fig. 8).

\section{EL TELESCOPIO DE WILLIAM HERSCHEL}

A esta pérdida como hemos visto reparable hay que añadir la desaparición del instrumento óptico más importante del Observatorio, el telescopio construido entre 1796 y 1797 por William Herschel (1738-1825), célebre astrónomo descubridor de Urano.(Figs. 9 y 10) Este gran telescopio, de 25 pies ingleses de longitud $(7,62 \mathrm{~m})$ y un espejo de 24 pulgadas de diámetro, fue en su momento uno de los mayores del mundo, y según su autor el de mayor calidad. ${ }^{14}$ Su reconstrucción es perfectamente posible gracias a la documentación utilizada para el montaje en Madrid, conservada en la biblioteca del propio Observatorio:

1. Un tomo de $88,66 \mathrm{~cm}$, titulado «Planos generales y particulares del telescopio de veinte y cinco pies ingleses de largo construidos en virtud de orden del Rey y disposicion del Ex. ${ }^{\text {mo }}$ S. ${ }^{\mathrm{R}}$ Principe de la Paz para la descripcion de dicho instrumento. Por D. ${ }^{\mathrm{n}}$ Josef de Mendoza Rios, miembro de la Real Sociedad de Londres». Contiene 12 acuarelas de 88,66 cm. Hay una edición facsímil reducida a la mitad, publicada por el Instituto Geográfico Nacional.

2. Un tomo de $31,20 \mathrm{~cm}$, en francés, titulado «Explication des plans du grand telescope de 25 pieds».

3. Un tomo de $64,50 \mathrm{~cm}$, en inglés, titulado «Eight drawings with references to the pieces of the 25 feet telescope packed in the cases specified in the bill of parcells». Contiene 8 acuarelas de $64,50 \mathrm{~cm}$.

4. Un cuaderno firmado por Mendoza, en inglés, titulado «Contents of the boxes in which the various parts of the 25 feet Herschelian telescope are packed up.».

5. Un cuaderno, en inglés, titulado «Iron, brass and other works belonging to the telescope».

6. Un cuaderno, en inglés, firmado por Herschel, titulado «Directions for the management of the telescope».

El telescopio construido por Herschel tuvo una existencia de apenas cuatro años, los transcurridos desde el montaje en 1804 en el Observatorio de Madrid hasta su destrucción en 1808 a causa de la invasión francesa, de la que únicamente pudieron salvarse los espejos:

«Dos espejos de metal, de 2 pies y medio de diámetro, señalados con los numeros 1 y 2 , en sus caxas de pino, pertenecientes al gran Telescopio de Herschell de 25 pies Ingleses de largo: es lo unico que se ha salvado de ésta asombrosa pieza: los franceses destruyeron y quemaron su maquinamento. ${ }^{15}$

Sobre una plataforma de fábrica se apoyaba una base circular de 30 pies de diámetro, constituida por 29 segmentos de roble. La estructura era de madera, y el tubo de hierro bati-

\footnotetext{
${ }_{13}$ «Descripción del edificio del Rl. Museo, por su autor D. Juan de Villanueva», en Arquitectura 304, Madrid, C.O.A.M. (1996), 115.

${ }^{14}$ LÓPEZ ARROYO, M.: El Gran Telescopio de W. Herschel, I. G. N., Observatorio Astronómico Nacional, Madrid, 1978, intr. y 9.

${ }^{15}$ «Lista de los Instrumentos del Observatorio Astronomico que se han trasladado al Rl Gabinete de Historia Natural», Museo Nacional de Ciencias Naturales, "Real Observatorio Astronómico de Madrid", caja 1, carpeta 2.
} 
do, de dos pies de diámetro y 25 de longitud. Existía además un pequeño observatorio auxiliar donde se encontraban los instrumentos necesarios para las observaciones.

No se conoce con certeza el lugar donde estaba situado este instrumento, aunque seguramente quedaría instalado fuera de los actuales límites del solar, ya que existe una referencia al traslado del cementerio, colocándose el telescopio en su lugar, "esto es, un poco más al sur de donde se halla actualmente [se hallaba] situada la casilla del inclinómetro."16

\section{¿JARDINES EN EL OBSERVATORIO ASTRONÓMICO?}

Hasta 1865, poco después de la llegada del agua del Canal de Isabel II en 1861, no se conoce la existencia de jardín alguno en el solar del Observatorio. ${ }^{17}$ Más bien al contrario, de la aguada de Velázquez parece desprenderse que la ausencia de jardín fue deliberada, como si ese aspecto desolado, casi cósmico, estuviera relacionado con la actividad realizada en el edificio, la astronomía.

La sutil integración entre el edificio y su entorno revela una sensibilidad extraordinariamente avanzada para su tiempo. A lo largo de toda su obra Villanueva utiliza los accidentes del terreno para realzar sus edificios, por lọ que cabe pensar que no hubiera deseado un jardín en sentido estricto para el Observatorio. Se utilizó seguramente un tipo de intervención extrañamente moderna, basado en la ordenación de la vegetación espontánea o subespontánea. Aquí y allá, como se observa en la aguada de Velázquez, se introducirían algunos árboles de sombra y frutales, y en general plantas de escasas exigencias.

El rasgo mas característico de los jardines actuales es la presencia de especies típicamente ruderales, como amapolas, avenas, malvas o rubia, lo que se traduce en una intensa sensación de abandono. Cabe destacar además la abundancia de la cambronera (Lycium europaeum L.), seguramente un resto de la vegetación natural. En la parte posterior del edificio principal se encuentra un magnífico ejemplar de albaricoquero, y es asimismo notable la presencia de árboles frecuentes en el Parque del Retiro, como la morera, el nogal, el olmo y el tilo.

La rehabilitación de los terrenos del Observatorio significaría una mejora evidente para esta institución. Sería recomendable la organización de una visita pública, que encajaría perfectamente dentro de las actuales ideas de la Dirección del Observatorio de convertir este recinto en un conjunto científico único y vivo. De este modo se gozaría en él de un ambiente parecido al que puede imaginarse a mediados del siglo pasado:

«Exmo. Señor.

Habiendome quedado solo en este establecimiento, según consta a S.E., desearia tubiera a bien ordenar de modo que un portero del Gabinete pasase todos los dias al observatorio durante la ausencia del director, p..$^{\text {a }}$ que tenga una persona que este en la puerta para abrir a multitud de personas inteligentes que en este tiempo van para ver los astros.

El año anterior visitaron una noche S.S. M.M. el establecimiento y me hallaba sin tener á quien mandar trasladase de una parte á otra los tripodes de los instrumentos que son demasiado pesados.

\footnotetext{
${ }^{16}$ LÓPEZ ARROYO, M.: El Gran Telescopio de W. Herschel, 13.

${ }^{17}$ TRABAJOS DEL PLANO DE MADRID/ DISTRITO DEL CONGRESO/ Parque del Retiro.- Jardín Botánico y Paseo del Prado, Dirección General del Instituto Geográfico y Estadístico, Sección de Reproducciones Fotomecánicas, ¿1865?, $440 \times 620 \mathrm{~mm}$.
} 
En este mismo dia le ha honrado el Sr. Infante Francisco, el cual ha quedado en volver para ver la Luna, la noche que se halle mas aproposito para el objeto.

En tales circunstancias Exmo. Señor creo me concedera lo que tan justamente pido.

Dios que a V.E. m. ${ }^{\mathrm{s}}$ a. ${ }^{\mathrm{s}}$ Madrid

3 de Julio de 1845

Jose Martinez / Exmo. Sr. Presidente del Museo de Ciencias Naturales.» ${ }^{18}$

En mi opinión la solución sería conjugar la reconstrucción parcial de la escena dibujada por Velázquez, es decir de la escena correspondiente a finales del XVIII, con algunos elementos correspondientes al siglo XIX que la prudencia aconseja conservar (a pesar del dudoso interés de algunas de estas construcciones), como la Casa de Astrónomos y los pabellones del astrógrafo y de los ecuatoriales Grubb. En todos los planos en los que aparece el Observatorio se observa como el límite sur de los terrenos no ha variado desde el siglo XVIII, lo que significa que esta rehabilitación es posible. ${ }^{19}$

Sin embargo, la recuperación aislada de la construcción de acceso carecería de sentido si no fuera acompañada de la creación de un itinerario de llegada y visita diferente del actual. No hay duda que la reconstrucción del gran telescopio de Herschel sería un sugestivo ingrediente en esta hipotética visita. Antes que la creación de un jardín formal, la rehabilitación del entorno del Observatorio debería consistir en mi opinión en la potenciación de una vegetación seminatural. En las partes no arboladas podrían disponerse especies xerofíticas como la cambronera, el esparto, la escoba, el romero o el tomillo, es decir las mismas que pueden encontrarse en cualquier cerro de la parte sur de Madrid. Todo ello encajaría perfectamente con la austera imagen dibujada por Velázquez. Finalmente, cabe imaginar la presencia de otras plantas también presentes en la parte sur del cercano Parque del Retiro, como el almendro, el árbol del amor, la encina, el fresno, el granado, la higuera, el nogal, el olmo o el tilo.

${ }^{18}$ Museo Nacional de Ciencias Naturales, "Real Observatorio Astronómico de Madrid”, caja 1, carpeta 21.

${ }^{19}$ Ver CABAÑAS DIAZ, J. C.: «Historia de los terrenos del Observatorio Astronómico de Madrid». 
1

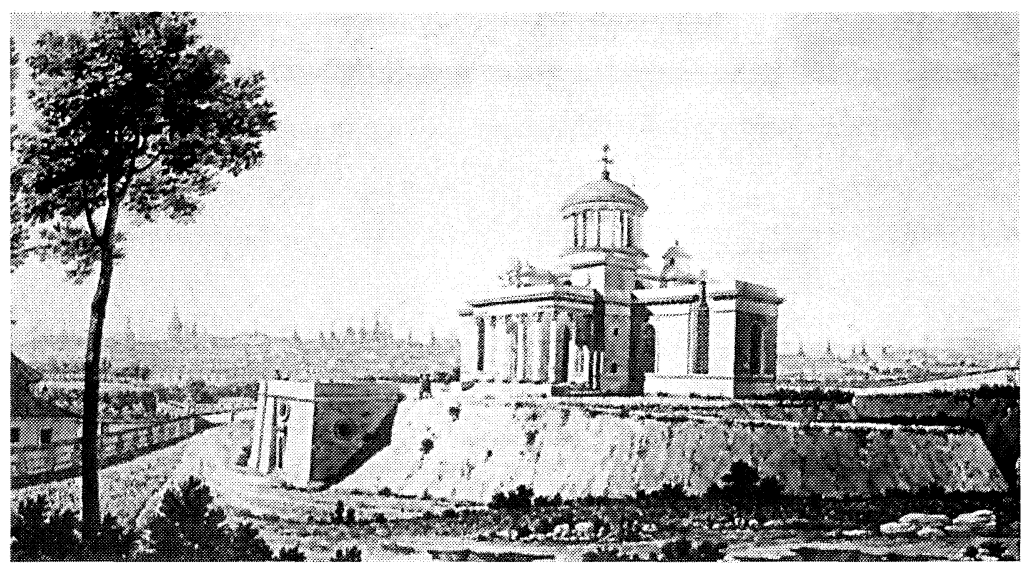

2
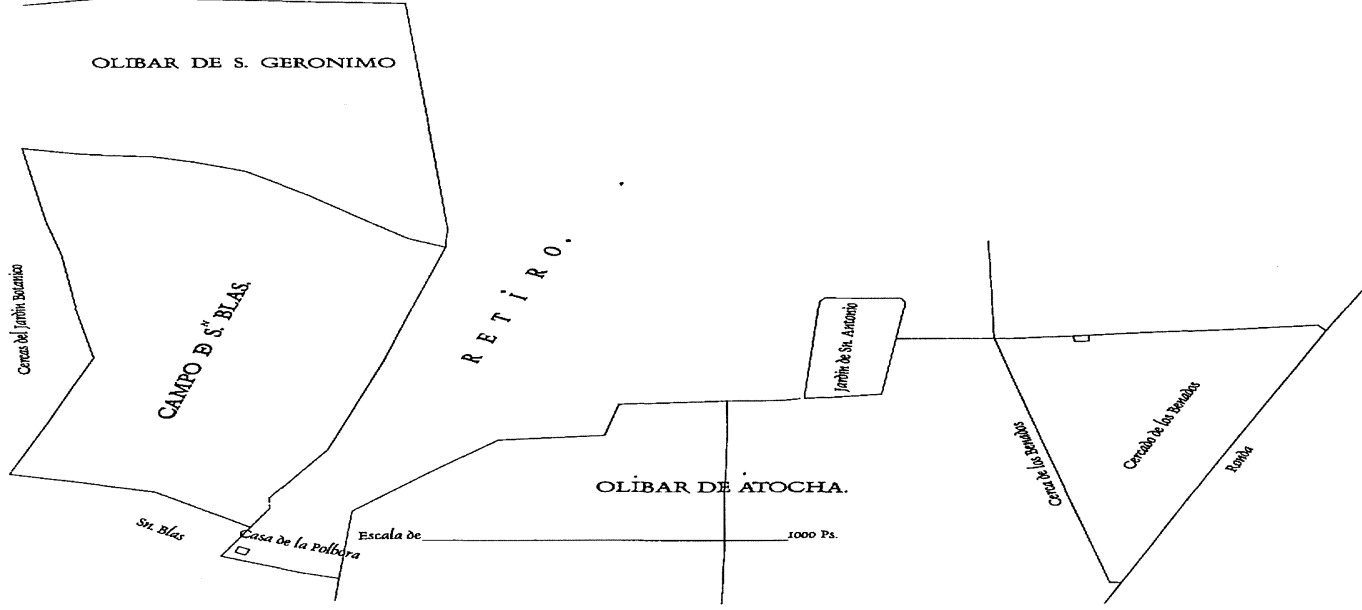

3

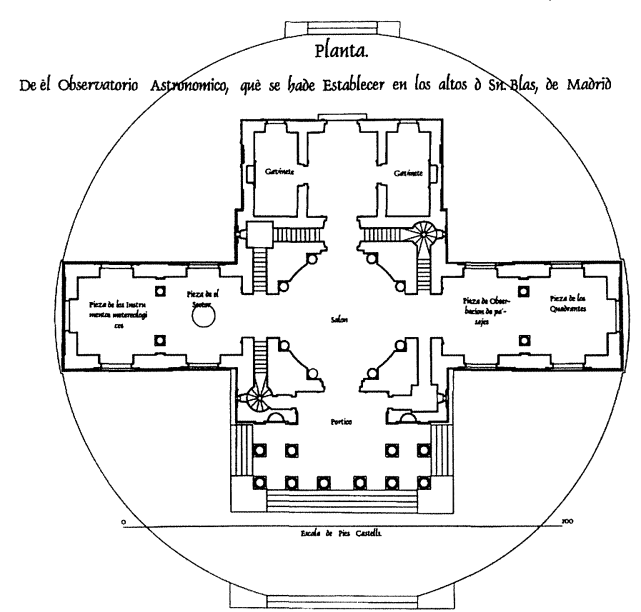

Fig. 1. Isidro Velázquez, Vista del Observatorio Astronómico hacia 1795. Dibujo a Lápiz, aguada de tinta china color sepia. Papel amarillento verjurado pegado a otro. 278 x $459 \mathrm{~mm}$. Firmado a lápiz: "I. Vlqz".

Fig. 2. ¿Juan de Villanueva?, "Plano del Real Sitio del Buen Retiro, de todas las tapias o cercas del retiro que lindan con el Olivar de Atocha, Jardín Botánico y Huerta de San Jerónimo”, ¿Madrid, 17 de marzo de 1785? Archivo del Palacio Real, plano 55, calco del autor.

Fig. 3. Planta original del Observatorio Astronómico de Juan de Villanueva, calco del autor. 

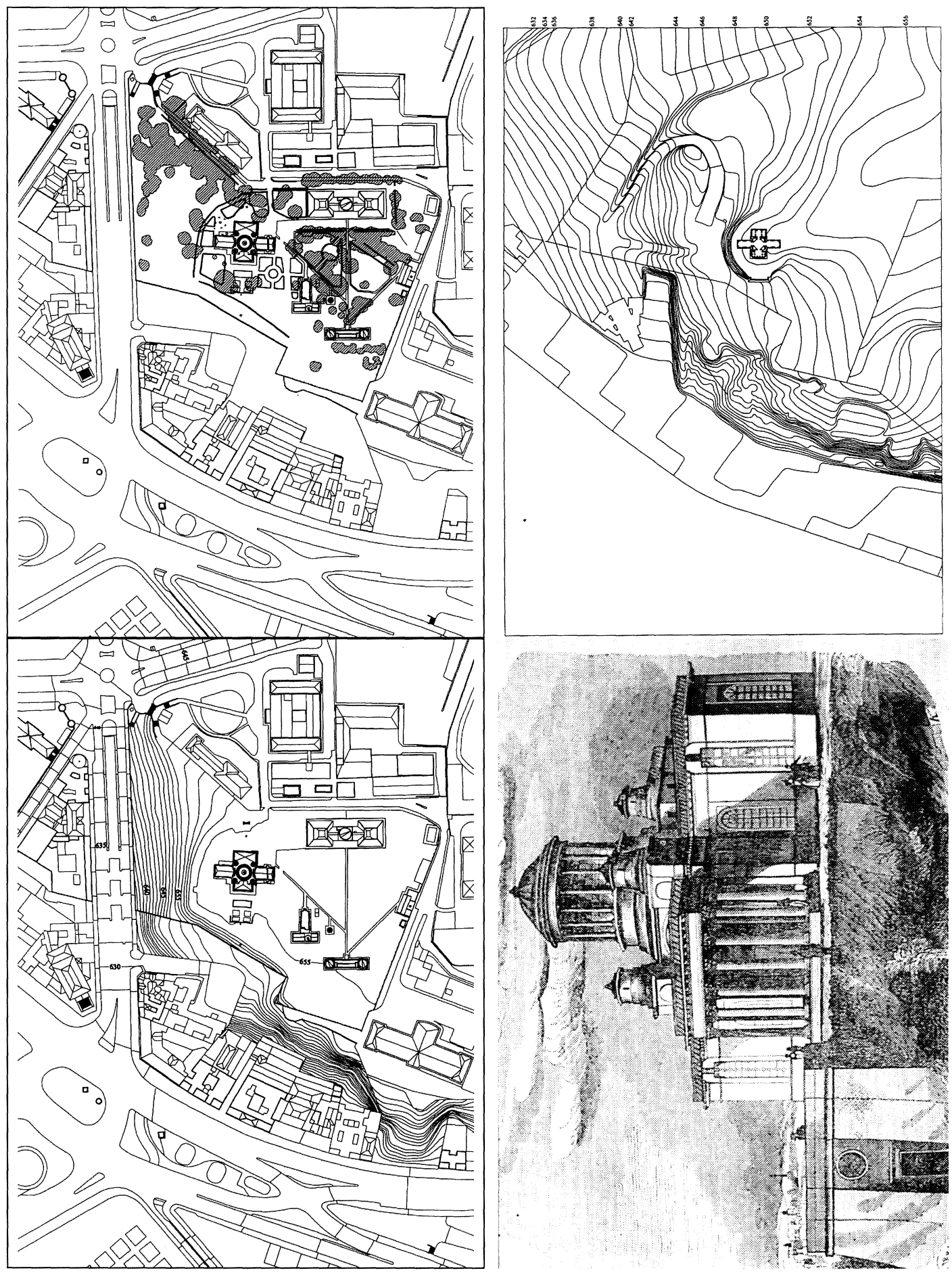

Fig. 4. Estado actual del observatorio Astronómico, dibujo de los Estudios Arabes de Granada (C.S.I.C.) y del autor.

Fig. 5. Topografía de la zona ObservatoriaAstronómica en 1872, calco del autor.

Fig. 6. Topografía de la zona del Observatorio Astronómico en la actualidad, calco del autor.

Fig. 7. "Observatorio Metereológico" En P. Madoz, Diccionario geográfico-estadístico-histórico de España y sus posesiones de ultramar, vol. X, p. 985, Madrid,d 1847. 

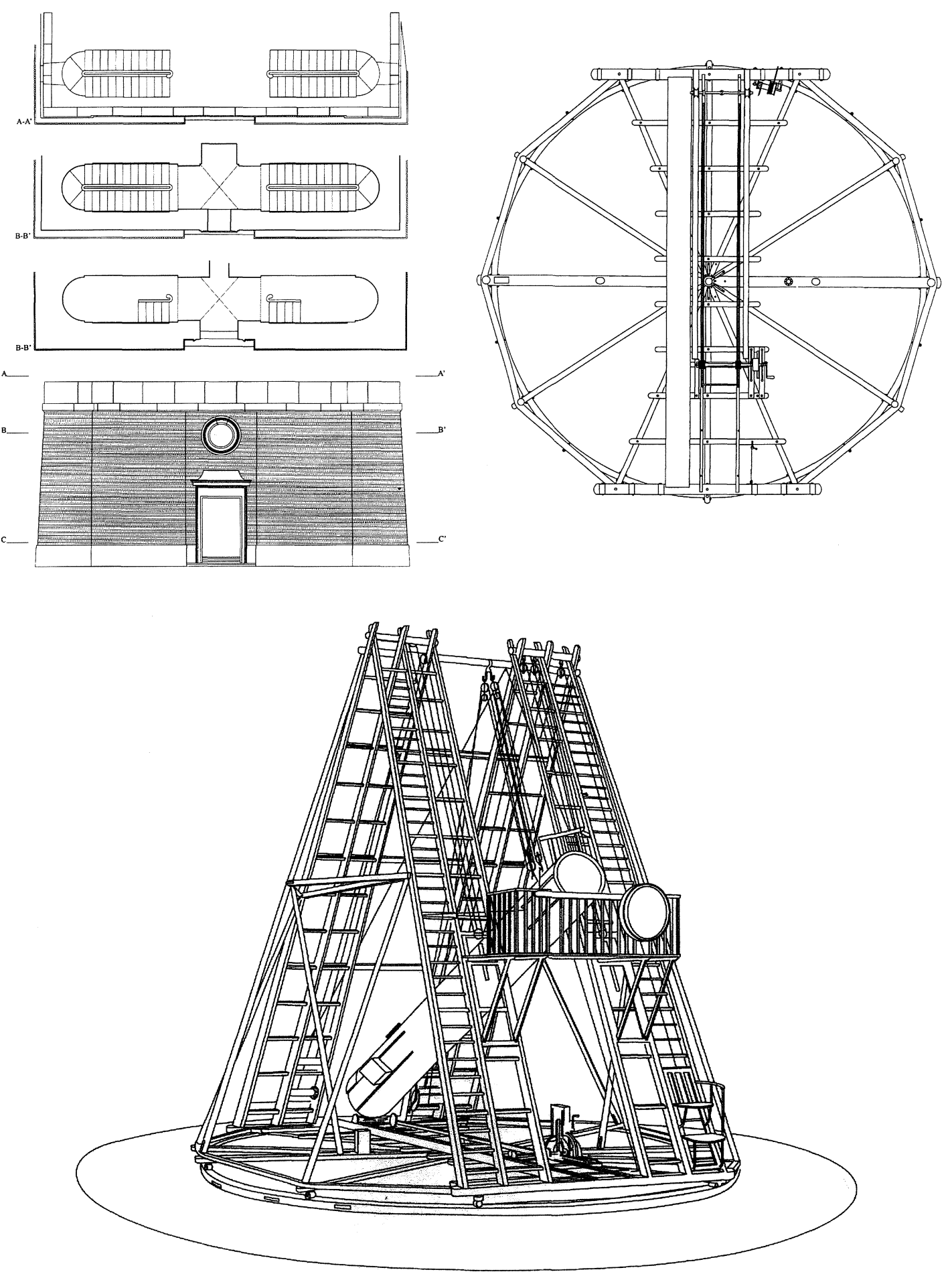

Fig. 8. La caja de escaleras, actual: dibujo del autor.

Fig. 9. Vista del telescopio con sus correspondientes aparatos, según el original de Mendoza Ríos, calco del autor.

Fig. 10. Plan general de la estructura del instrumento, según el original de Mendoza Ríos, calco del autor. 